\title{
Estradiol triggers sonic-hedgehog-induced angiogenesis during peripheral nerve regeneration by downregulating hedgehog-interacting protein
}

\author{
Haruki Sekiguchi ${ }^{1,2}$, Masaaki li ${ }^{1,3}$, Kentaro Jujo ${ }^{1,2}$, Marie-Ange Renault ${ }^{1,4,5}$, Tina Thorne ${ }^{1}$, Trevor Clarke ${ }^{1}$, Aiko Ito ${ }^{1}$, \\ Toshikazu Tanaka ${ }^{1,6}$, Ekaterina Klyachko', Yasuhiko Tabata7 ${ }^{7}$ Nobuhisa Hagiwara ${ }^{2}$ and Douglas Losordo ${ }^{1}$
}

Both estradiol (E2) and Sonic Hedgehog (Shh) contribute to angiogenesis and nerve regeneration. Here, we investigated whether E2 improves the recovery of injured nerves by downregulating the Shh inhibitor hedgehog-interacting protein (HIP) and increasing Shh-induced angiogenesis. Mice were treated with local injections of E2 or placebo one week before nerve-crush injury; 28 days after injury, nerve conduction velocity, exercise duration, and vascularity were significantly greater in E2-treated mice than in placebo-treated mice. E2 treatment was also associated with higher mRNA levels of Shh, the Shh receptor Patched-1, and the Shh transcriptional target Gli1, but with lower levels of HIP. The E2-induced enhancement of nerve vascularity was abolished by the Shh inhibitor cyclopamine, and the effect of E2 treatment on Shh, Gli1, and HIP mRNA expression was abolished by the E2 inhibitor ICl. Gli-luciferase activity in human umbilical-vein endothelial cells (HUVECs) increased more after treatment with E2 and Shh than after treatment with E2 alone, and E2 treatment reduced HIP expression in HUVECs and Schwann cells without altering Shh expression. Collectively, these findings suggest that E2 improves nerve recovery, at least in part, by reducing HIP expression, which subsequently leads to an increase in Shh signaling and Shh-induced angiogenesis.

Laboratory Investigation (2012) 92, 532-542; doi:10.1038/labinvest.2012.6; published online 13 February 2012

KEYWORDS: angiogenesis; estradiol; hedgehog-interacting protein; nervous system; sonic hedgehog

The physiological effects of estrogen extend well beyond its role in development and menstrual-cycle regulation. Estrogen exists in multiple forms: estrone sulfate, which is inactive and has a relatively long half-life in peripheral blood; estrone, the active product of estrone sulfate metabolism; and estradiol (E2), the active product of estrone metabolism by $17 \beta$-hydroxysteroid dehydrogenase type 1 . Because serum E2 levels are inversely correlated with the onset of cardiovascular disease in postmenopausal women, ${ }^{1}$ E2 was investigated for the prevention of cardiovascular disease in several clinical trials of hormone replacement therapy. ${ }^{2,3}$

Evidence indicates that E2 reduces the risk of cardiovascular events in younger postmenopausal women. ${ }^{4,5}$ The favorable effects of E2 may be partly attributed to enhanced angiogenesis: functional estrogen receptors are essential for the induction of angiogenesis by basic fibroblast growth factor, ${ }^{6}$ and estrogen accelerates endothelial recovery by increasing nitric oxide production and enhancing the mobilization of bone marrow-derived endothelial progenitor cells. $^{7}$ E2 also appears to contribute to the preservation and recovery of injured nerve tissue: the expression of estrogen receptors $\alpha$ and $\beta$ increases after nerve injury, ${ }^{8}$ and implantation of E2-containing pellets one week before nervecrush injury stimulated nerve preservation/regeneration, ${ }^{9}$ perhaps by activating mitogen-activated protein kinase signaling. ${ }^{8}$ E2 also protects against ischemia-induced neural death by activating the Akt pathway. ${ }^{10}$

\footnotetext{
${ }^{1}$ Feinberg Cardiovascular Research Institute and Program in Cardiovascular Regenerative Medicine, Division of Cardiovascular Medicine, Department of Medicine, Northwestern University Feinberg School of Medicine and Northwestern Memorial Hospital, Chicago, IL, USA; ${ }^{2}$ Department of Cardiology, Tokyo Women's Medical University, Tokyo, Japan; ${ }^{3}$ Group of Translational Stem Cell Research, Department of Pharmacology, Osaka Medical College, Osaka, Japan; ${ }^{4}$ Université de Bordeaux, Adaptation cardiovasculaire à l'ischémie, Pessac, France; ${ }^{5}$ INSERM, U1034, Adaptation cardiovasculaire à l'ischémie, Pessac, France; ${ }^{6}$ University of Pittsburgh Medical Center, Pittsburgh, PA, USA and ${ }^{7}$ Department of Biomaterials, Field of Tissue Engineering, Institute for Frontier Medical Sciences, Kyoto University, Kyoto, Japan Correspondence: Dr M li, MD, PhD, Group of Translational Stem Cell Research, Department of Pharmacology, Osaka Medical College, 2-7, Daigaku-machi, Takatsuki, Osaka 569-8686, Japan.
}

E-mail: masa0331@mac.com

Received 16 May 2011; revised 1 December 2011; accepted 14 December 2011 
Recent findings suggest that Sonic Hedgehog (Shh), a member of the mammalian hedgehog protein family, may also contribute to nerve regeneration and repair. The hedgehog proteins were first characterized in Drosophila as regulators of segmental polarity during development. ${ }^{11}$ In vertebrate development, Shh regulates the patterning of the central nervous system, ${ }^{12-14}$ whereas the activity of another hedgehog protein, Desert Hedgehog, is restricted to the peripheral nervous system and appears to influence cellular elements of the epineural and perineural sheath. ${ }^{15}$ In the absence of injury, Shh signaling is attenuated, at least in part, by interactions between Shh and the Shh inhibitor hedgehoginteracting protein (HIP); ${ }^{16}$ however, Hashimoto et al ${ }^{17}$ have shown that the expression of Shh increases after peripheralnerve injury in adult rats, and that cyclopamine, which disrupts hedgehog signaling, reduces the survival of motor neurons. Furthermore, exogenous Shh protein administration has been shown to enhance angiogenesis and improve nerve function in a diabetic neuropathy model. ${ }^{18}$

Collectively, these observations indicate that both E2 and Shh may contribute to the neovascularization and regeneration of injured peripheral nerves in adult animals, and estrogen has recently been linked to the upregulation of Shh expression in breast-cancer cells. ${ }^{19}$ Here, we investigated whether E2 improves functional recovery in mechanically injured nerves by activating the hedgehog-signaling pathway.

\section{MATERIALS AND METHODS}

\section{Animal Models and Treatments}

Experiments were performed in ovariectomized female wild-type (C57BL/6J) mice and in ovariectomized female heterozygous Patched-1(Ptch1)-LacZ (NLS-Ptch1-lacZ) or heterozygous Gli1-LacZ (NLS-Gli1-lacZ) mice and their wild-type littermates (The Jackson Laboratory, Bar Harbor, ME, USA) E2 in poly lactide-co-glycolide (PLGA) was kindly provided by Dr. Tabata (Kyoto University, Japan). All surgical procedures were approved by the Institutional Animal Care and Use Committee of Northwestern University's Feinberg School of Medicine and were consistent with the Guide for the Care and Use of Laboratory Animals published by the United States National Institutes of Health. ${ }^{20}$ Ovariectomy was performed 2 weeks before injury and E2-PLGA, saline-PLGA, or E2 pellets (Innovative Research of America, Sarasota, FL, USA) were administered at the designated injury sight 1 week later (ie, 1 week before injury); E2-PLGA and saline PLGA were administered via subcutaneous injection, and the E2 pellets were subcutaneously implanted. Cyclopamine $(50 \mathrm{mg} /$ $\mathrm{kg}$ per day) and the estrogen receptor antagonist ICI $182,780^{21}$ (8.3 mg/kg per day; Tocris Cookson, Bristol, UK) were intraperitoneally injected once daily from 2 days (cyclopamine) or 3 days (ICI) before injury until the time of killing. Serum E2 levels were determined with an E2-ELISA kit (Cayman Chemical, Ann Arbor, MI, USA) as directed by the manufacturer's instructions. Nerve-crush injury was induced as described previously ${ }^{22}$ and as summarized in the Supplementary Methods.

\section{Nerve Function}

Motor-nerve conduction velocity (MCV) and the duration of rotarod exercise were measured as summarized in the Supplementary Methods. MCV measurements in the injured limb were normalized to measurements performed in the uninjured contralateral limb. For exercise duration, the maximum of three measurements was reported for each mouse.

\section{Vascularity and Capillary Density}

Functional vascular structures were identified by injecting mice with fluorescein isothiocyanate-conjugated Bandeiraea simplicifolia-1 lectin (Vector Laboratories, Burlingame, CA, USA) 15 minutes before killing, and endothelial cells were identified by staining sections with anti-CD31 antibodies (BD Biosciences, San Jose, CA, USA). Capillary density was evaluated by counting double-positive tubular structures in five sections MAPK from the proximal, middle, and distal regions of both the injured and uninjured contralateral nerve (totaling 15 sections per nerve); three fields were evaluated in each section. Measurements in the injured nerve were normalized to those obtained in the uninjured nerve.

\section{Gene Expression}

For in-vivo assessments, the mRNA expression of Shh, smoothened (smo), Ptch1, Gli1, HIP, and vascular endothelial growth factor (VEGF) was evaluated via quantitative, real-time, reverse-transcriptase, polymerase chain reaction (qRT-PCR). Activation of Ptch1 and Gli1 expression in sections from Ptch1-LacZ and Gli1-LacZ mice was evaluated via $X$-gal staining. Ptch1 protein and HIP protein were identified with anti-Ptch1 and anti-HIP antibodies (Santa Cruz Biotechnology, Santa Cruz, CA, USA), endothelial cells were identified with anti-CD31 antibodies, and Schwann cells were identified with anti-S100 antibodies (Sigma-Aldrich, St Louis, MO, USA). Positively stained cells were counted in three sections from both the injured nerve and the uninjured contralateral nerve, three high-power fields per section; measurements in the injured nerve were normalized to those obtained in the uninjured nerve. For in-vitro assessments, human umbilical-vein endothelial cells (HUVECs) (Cascade Biologics, Portland, OR, USA), Schwann cells (SW10 cells; ATCC, Manassas, VA, USA), and NIH3T3 fibroblasts (ATCC, Manassas, VA, USA) were cultured in $1 \times 10^{-8} \mathrm{~mol} / 1$ E2 for $6 \mathrm{~h}$, then Shh, Ptch1, Gli1, and HIP mRNA expression were evaluated via qRT-PCR. Gli transcriptional activation in endothelial cells was evaluated via the Gli-luciferase assay. qRT-PCR and the Gli-luciferase assay were performed as summarized in the Supplementary Methods; qRT-PCR primer and probe sequences are provided in the Supplementary Table. 


\section{Statistical Analysis}

All values are reported as mean \pm s.e.m. Statistical analyses were performed with commercially available software (StatViewTM, Abacus Concepts, Berkeley, CA, USA); comparisons between two groups were tested for significance with the Mann-Whitney $U$-test, and comparisons between multiple groups were tested for significance via analysis of variance followed by post-hoc testing with the Tukey procedure. A $P$ value of $<0.05$ was considered significant.

\section{RESULTS}

Local E2 Injection Improves Nerve Functional Recovery and Vascularity after Nerve-Crush Injury without Significantly Increasing Serum Estrogen Levels

Because of the side effects and potential health risks associated with systemically administered E2, we investigated whether the apparent benefits of systemic E2, administered as an implanted E2 pellet, ${ }^{9}$ could be achieved with locally delivered E2. One week before surgical sciatic nerve-crush injury was induced in mice, E2 in PLGA or PLGA alone (placebo) was locally injected, or an extended-release E2 pellet was subcutaneously implanted, at the designated injury site. Local E2 administration did not significantly alter serum E2 levels, whereas serum E2 levels increased approximately 10-fold after pellet implantation (Supplementary Figure 1).

Functional recovery and motor coordination was monitored via MCV measurements performed at weekly intervals for 28 days after injury and by assessing the duration of rotarod exercise on day 28. MCV in all treatment groups was similar before nerve-crush injury and undetectable immediately afterward. MCV had improved in both E2-treatment groups on day 7 after injury, but remained undetectable in mice administered placebo, and was significantly higher in E2-treated mice than in placebo-treated mice at all subsequent time points (Figure 1a). On day 28 after injury, MCV approached pre-injury levels in both E2-treatment groups $(P<0.01)$, and the duration of rotarod exercise was significantly longer in E2-treated mice than in mice administered placebo $(P<0.05$; Figure $1 \mathrm{~b}) . \mathrm{MCV}$ and rotarod exercise measurements after E2 injection or pellet implantation did not differ significantly at any time point.

Nerve vascularity was assessed in both the injured and uninjured hind limbs of mice killed 28 days after nerve-crush injury. As observed in previous reports, ${ }^{23}$ nerve-crush injury alone increased vascularity, even in placebo-treated limbs. Both E2 injection and E2 pellet implantation enhanced the vasculogenic response (Figure 1c), and capillary density was significantly higher $(P<0.01)$ in the injured limbs of E2-treated mice than in the injured limbs of the placebotreatment group (Figure 1d); capillary density was also significantly higher $(P<0.01)$ when $\mathrm{E} 2$ was administered as a local injection rather than an implanted pellet.

Collectively, these observations indicate that both injected E2-PLGA and the implanted E2 pellets improve MCV, functional recovery, and nerve vascularity after injury, but the
E2-PLGA injections did not lead to the significant (and potentially harmful) increases in systemic estrogen levels observed after pellet implantation. Thus, E2 was administered via subcutaneous injection, rather than pellet implantation, in subsequent experiments.

\section{Local E2 Injection Enhances Shh-Pathway Activation after Nerve-Crush Injury}

The Shh pathway is activated by nerve-crush injury ${ }^{17}$ and contributes to vascular regeneration in response to ischemic injury. ${ }^{23,24}$ To determine whether the E2-induced enhancement of nerve function and vascularity after injury could have occurred through elevated Shh signaling, we monitored the mRNA expression of Shh; smo, which activates downstream components of the hedgehog pathway; the Shh receptor Ptch1; the Shh transcriptional target Gli1; and VEGF, which has been linked to Shh signaling in a murine diabetic neuropathy model. ${ }^{18}$ Injured nerves had been pretreated with local injections of E2 or placebo 1 week before injury. In the absence of injury, E2 did not alter mRNA levels of Shh (E2: $0.26 \pm 0.12$, placebo: $0.23 \pm 0.12$; normalized to $18 \mathrm{~S}$ rRNA levels), Gli1 (E2: $59.1 \pm 12.0$, placebo: $60.5 \pm 9.4$ ), or VEGF (E2: $5.27 \pm 2.50$, placebo: $6.35 \pm 4.70$ ), and treatment of the injured limb did not alter Shh, Ptch1, smo, Gli1, or VEGF expression in the uninjured limb.

Shh expression was significantly elevated in the injured nerves of placebo-treated mice within $3 \mathrm{~h}$ of injury $(P<0.02)$ and continued to increase through hour 24 before returning to non-injury levels on day 7 (Figure 2a). Shh expression was even higher in the injured limbs of E2-treated mice $(P<0.01$ $v s$ placebo treatment from hour 6 through hour 12), but the time course of elevated Shh expression was similar in both groups. The mRNA expression of smo declined after injury but was similar in E2-treated and placebo-treated nerves at all time points (Figure 2b). Ptch1 and Glil expression declined in the injured nerves of placebo-treated mice through hours 6 (Ptch1) and 24 (Gli1) after injury, then slowly recovered to approximately 40\% (Ptch1) and 75\% (Gli1) of non-injury levels on day 7 (Figures $2 \mathrm{c}$ and d). The initial declines in Ptch1 and Gli1 expression were also observed in the injured nerves of E2-treated mice, but recovery was enhanced: Ptch1 expression was significantly higher $(P<0.01)$ from day 3 through day 7 , and Gli1 expression was significantly higher $(P<0.01)$ from hour 24 through day 3, in E2-treated nerves than in placebo-treated nerves. E2 treatment was also associated with significantly higher mRNA levels of VEGF from hour 24 through day 3 (Figure 2e).

The apparent E2-induced enhancement of Shh signaling was corroborated with assessments performed in the injured and uninjured contralateral nerves of mice treated with E2 and the estrogen-receptor antagonist ICI. Three days after injury, Shh expression was significantly lower in nerves treated with both E2 and ICI than in nerves treated with E2 alone, whereas Shh expression after placebo treatment or E2-ICI co-administration was similar (Figure 2f). 


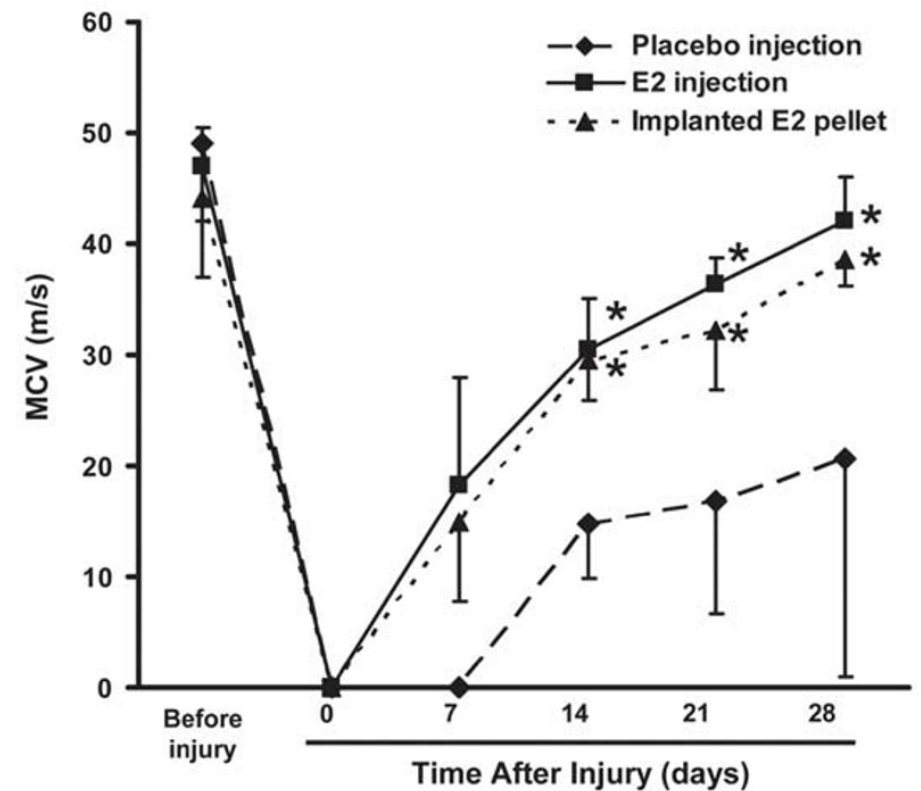

C
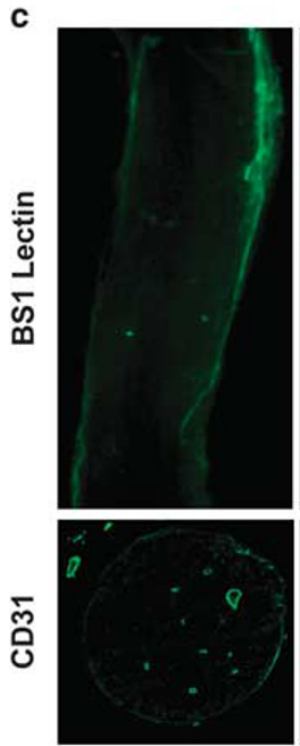

Uninjured
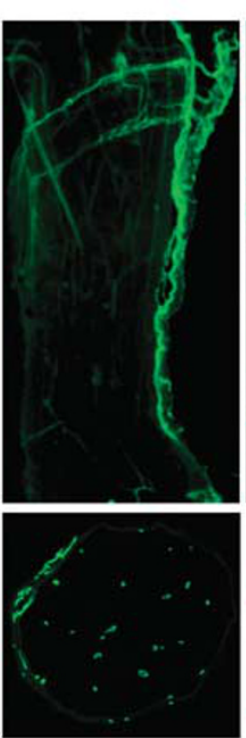

Placebo
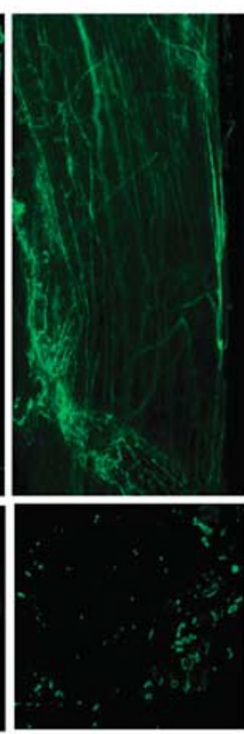

E2 injection
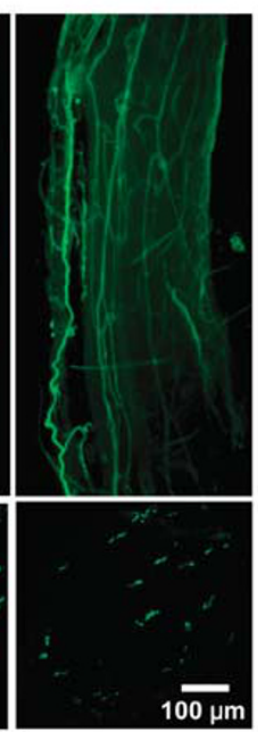

E2 pellet
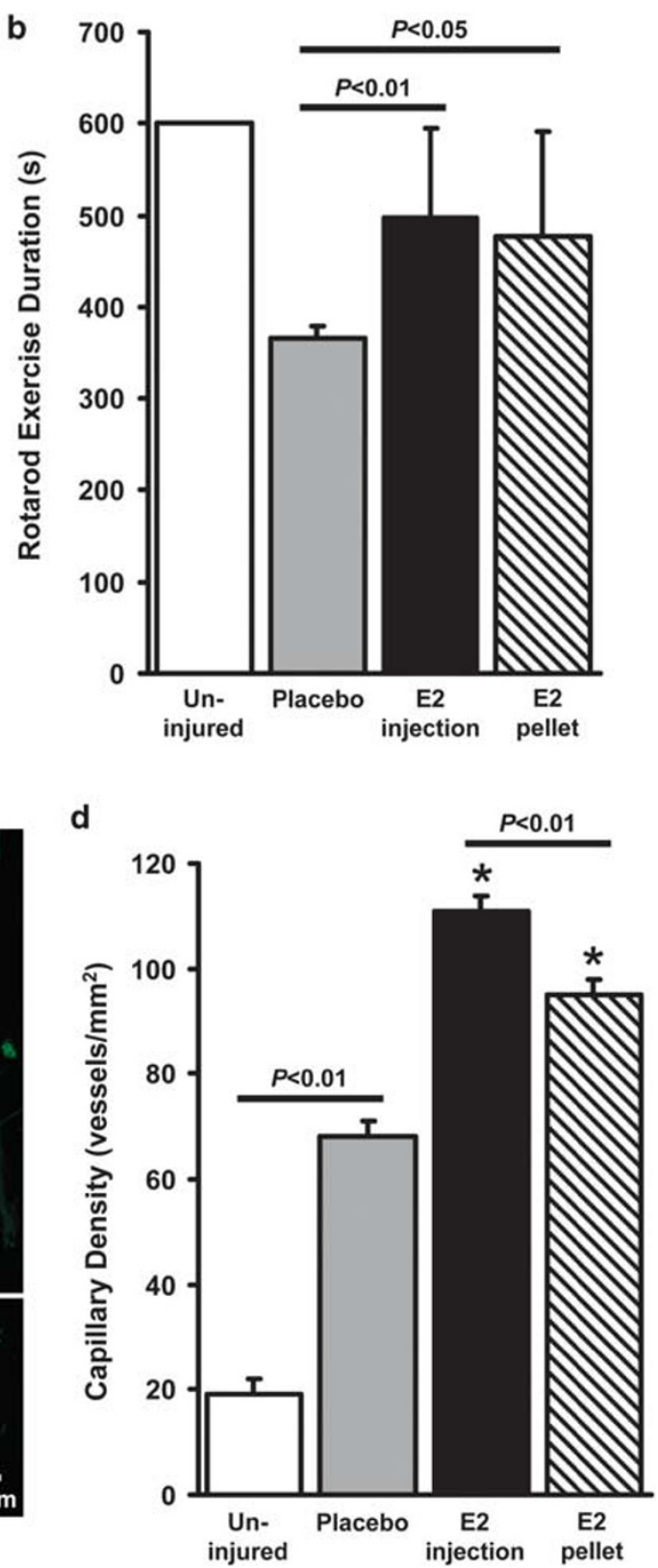

Figure 1 Local E2 injection improves the functional recovery and vascularity of injured nerves. One week before surgical sciatic nerve-crush injury, E2 $(100 \mu \mathrm{g})$ in PLGA (to ensure extended E2 delivery) or PLGA alone (placebo) was locally injected into the designated injury site, or an extended-release E2 pellet ( $0.5 \mathrm{mg}$ delivered over 60 days) was subcutaneously implanted into the limb. (a) MCV was measured before injury, immediately afterward (day 0 ), and at weekly intervals for the next 28 days; for clarity, only one error bar is shown per data point. (b) The duration of rotarod exercise was measured 28 days after injury; the duration for the uninjured group was equal to the length of the experiment (600 s, s.e.m. $=0$ ). (c) Functional vasculature was identified in sections of injured nerve tissue by injecting mice with fluorescein-conjugated BS-1 lectin 15 minutes before killing on day 28 . (d) Capillary density was evaluated by staining cross sections of the vasa nervorum from mice killed on day 28 with the endothelial-cell marker $C D 31 ;{ }^{\star} P<0.01$ vs placebo.

\section{Local E2 Injection Activates Ptch1 and Gli1 Protein Expression in the Injured Nerve}

To confirm that the E2-induced enhancement of Ptch1 and Gli1 mRNA expression after nerve-crush injury was accompanied by the activation of protein expression, experiments were performed in mice that carried nondisruptive insertions of a lacZ reporter gene upstream of the Ptch1- (Ptch1-LacZ mice) or the Gli1- (Gli1-LacZ mice) coding region. Assess- ments were performed 3 days after injury, when Ptch1, Gli1, and VEGF mRNA expression were higher in the E2-treated nerves than in the placebo-treated nerves of wild-type mice. In Ptch1-LacZ mice, Xgal staining of whole-mount sciatic nerve tissue found markedly greater evidence of Ptch1 expression at the site of nerve-crush injury in E2-treated nerves than in placebo-treated nerves (Figures $3 a$ and $b$ ). In Gli1-LacZ mice, LacZ-expressing cells were significantly more 
common in both the endoneurium and the perineurium of E2-treated nerves than in the corresponding regions of uninjured nerves $(P<0.01)$, whereas only the endoneurium of placebo-treated nerves displayed evidence of Gli1 upregulation $(P<0.01$ vs uninjured nerves; Figures $3 \mathrm{c}$ and $\mathrm{d})$; LacZ expression in both regions was significantly more common after E2 treatment than after placebo treatment $(P<0.01)$. The expression of Gli1 mRNA was also elevated in E2-treated nerves $(P<0.01$ vs placebo treatment), and this enhancement was abolished by co-treatment with ICI (Figure 3e). In sections co-stained with CD31 antibodies, X-gal-CD31 double-positive cells were observed in E2-treated nerves but not in placebotreated nerves (Figure 3f). Thus, E2 appears to enhance Shh signaling in endothelial cells after nerve-crush injury.

\section{The E2-Induced Enhancement of Vascularity after Nerve- Crush Injury Requires Shh-Pathway Activation}

To determine whether the E2-induced increase in nerve vascularity after crush injury could be attributed to enhanced Shh signaling, functional nerve vascularity and capillary density were assessed in the injured nerves of Ptch1-LacZ mice treated with placebo, with E2, with cyclopamine, which
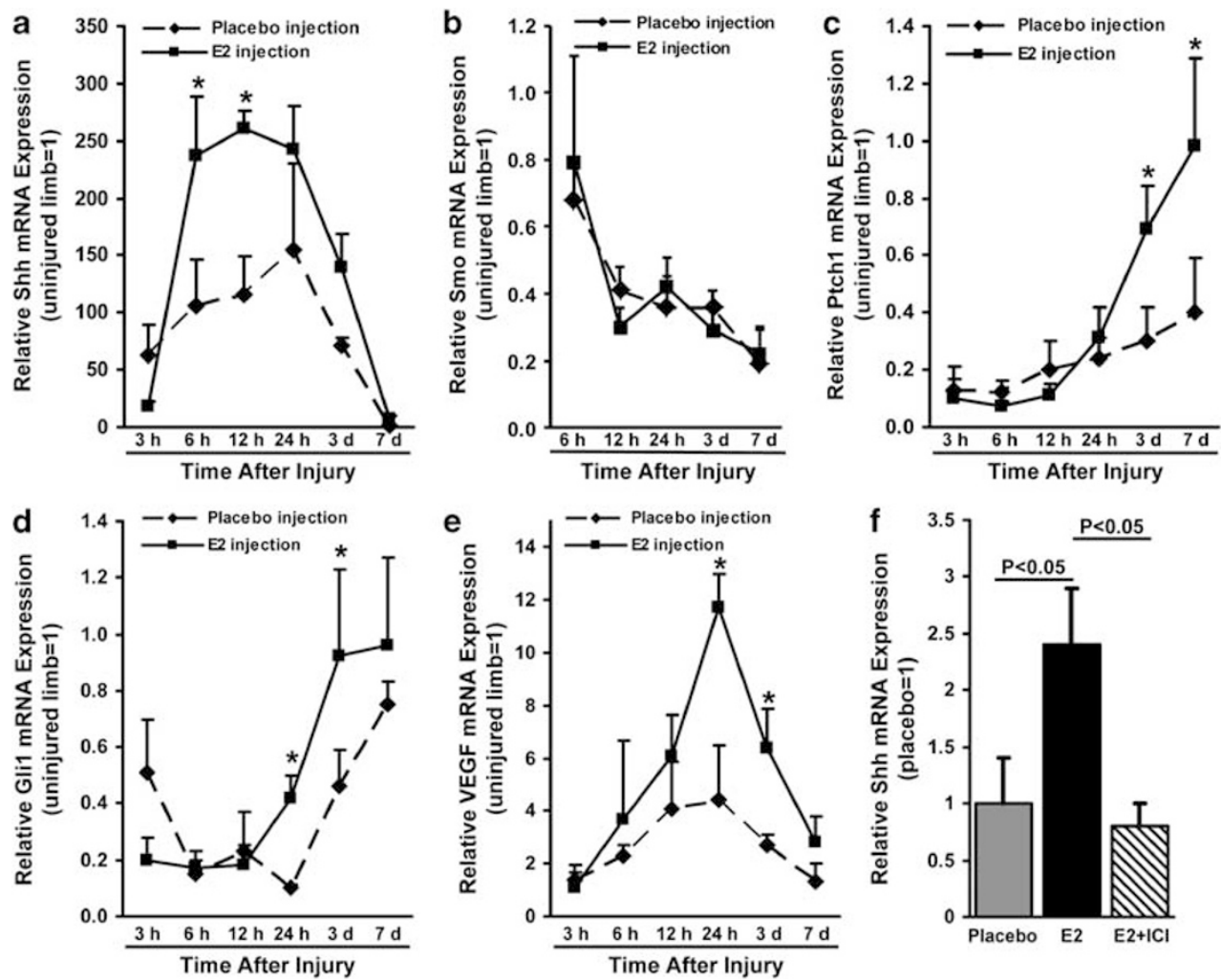

Figure 2 Local E2 injection enhances Shh-pathway activation after nerve injury. One week before surgical sciatic nerve-crush injury, E2 or saline (placebo) in PLGA was locally injected into the designated injury site. The mRNA expression of (a) Shh, (b) smo, which activates downstream components of the hedgehog signaling pathway, (c) the Shh receptor Ptch1, (d) the Shh transcriptional target Gli1, and (e) VEGF was evaluated for up to 7 days after injury via qRT-PCR; measurements were performed in both the injured and uninjured contralateral nerves, normalized to endogenous $18 \mathrm{~S}$ rRNA levels, and presented relative to the values obtained in the uninjured limb; for clarity, only one error bar is shown per data point. (f) Shh mRNA expression was evaluated after nerve-crush injury in mice treated with placebo, with E2 injections, or with injections of E2 and the E2-receptor blocker ICl; ICl (8.3 mg/kg) was injected intraperitoneally 3 days before injury, and assessments were performed 3 days after injury, when both Ptch1 and Gli1 mRNA expression were significantly upregulated (c, d), via qRT-PCR, normalized to endogenous $18 \mathrm{~S}$ rRNA levels, and presented relative to the values obtained in placebo-treated mice. ${ }^{*} P<0.01$.

Figure 3 Local E2 injection activates Ptch1 and Gli1 expression in injured nerves. Ptch1-LacZ mice and Gli1-LacZ mice received local injections of E2 or saline (placebo) and PLGA 1 week before sciatic nerve-crush injury; subsequent assessments were performed in mice killed 3 days after injury, when both Ptch1 and Gli1 mRNA expression were significantly upregulated in wild-type mice (Figures 2c and d). (a, b) The activation of Ptch1-LacZ protein expression was evaluated by staining (a) whole-mount tissues and (b) cross sections of the injured nerves from Ptch1-LacZ mice with X-gal (blue). (c, d) The activation of Gli1-LacZ expression was evaluated in X-gal-stained sections of (c) epineural and (d) endoneural tissue from the injured nerves of Gli1-LacZ mice. (e) Gli1 mRNA expression was evaluated in Gli1-LacZ mice treated with placebo, with E2 injections, or with injections of E2 and the E2-receptor blocker $\mathrm{ICl}$; measurements were performed via qRT-PCR, normalized to endogenous $18 \mathrm{~S}$ rRNA levels, and presented relative to the values obtained in placebo-treated mice. (f) X-gal-stained sections from uninjured limbs and from the injured limbs of placebo-treated and E2-treated mice were co-stained for expression of the endothelial-specific marker CD31 (brown) to identify endothelial cells that expressed Gli1 (blue). ${ }^{\star} P<0.01$ vs uninjured; ${ }^{\dagger} P<0.01$ vs placebo or E2 $+\mathrm{ICI}$. 
blocks hedgehog signaling by interfering with smo, or with E2 and cyclopamine. Twenty-eight days after injury, both vascularity and capillary density were greater in E2-treated nerves than in placebo-treated nerves, but this enhancement was not observed in mice treated with both E2 and cyclo- pamine (Figures $4 \mathrm{a}$ and $\mathrm{b}$ ). Cyclopamine also abolished the E2-induced enhancement of Ptch1 upregulation in Ptch1LacZ mice (Figure 4c) and the E2-induced enhancement of Gli1 upregulation in Gli1-LacZ mice (Figure 4d), which confirms that the loss of E2-induced capillary growth in mice a

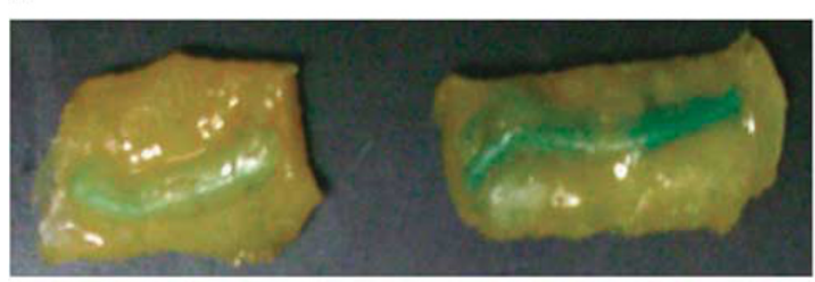

Placebo

c

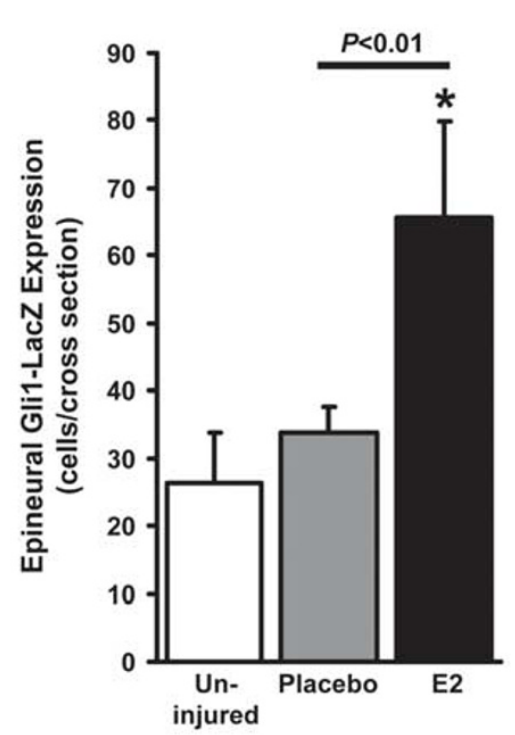

E2

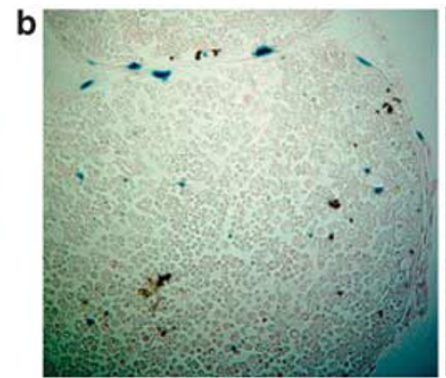

Placebo

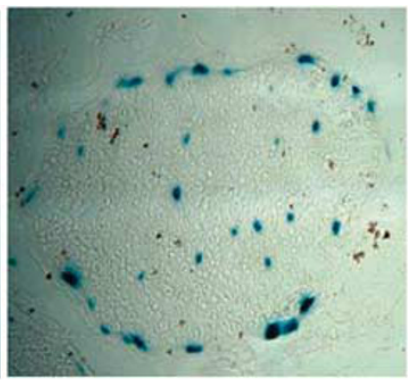

E2 d

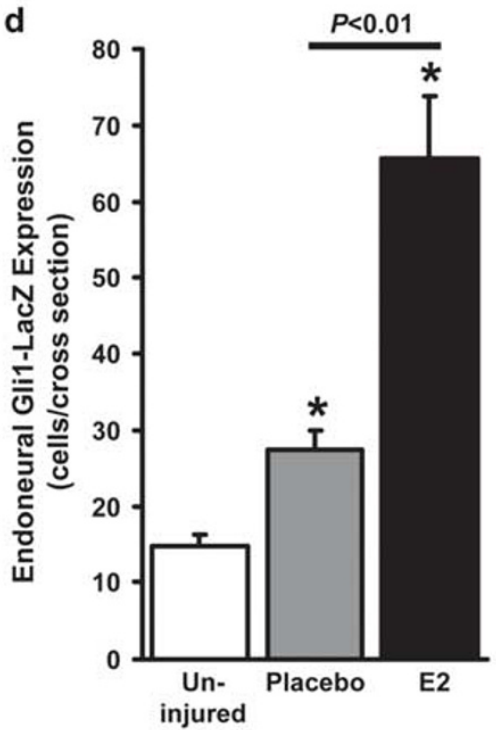

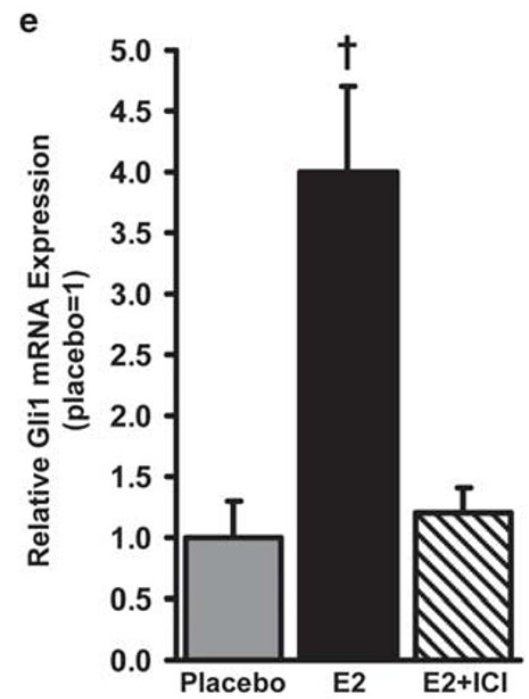

f

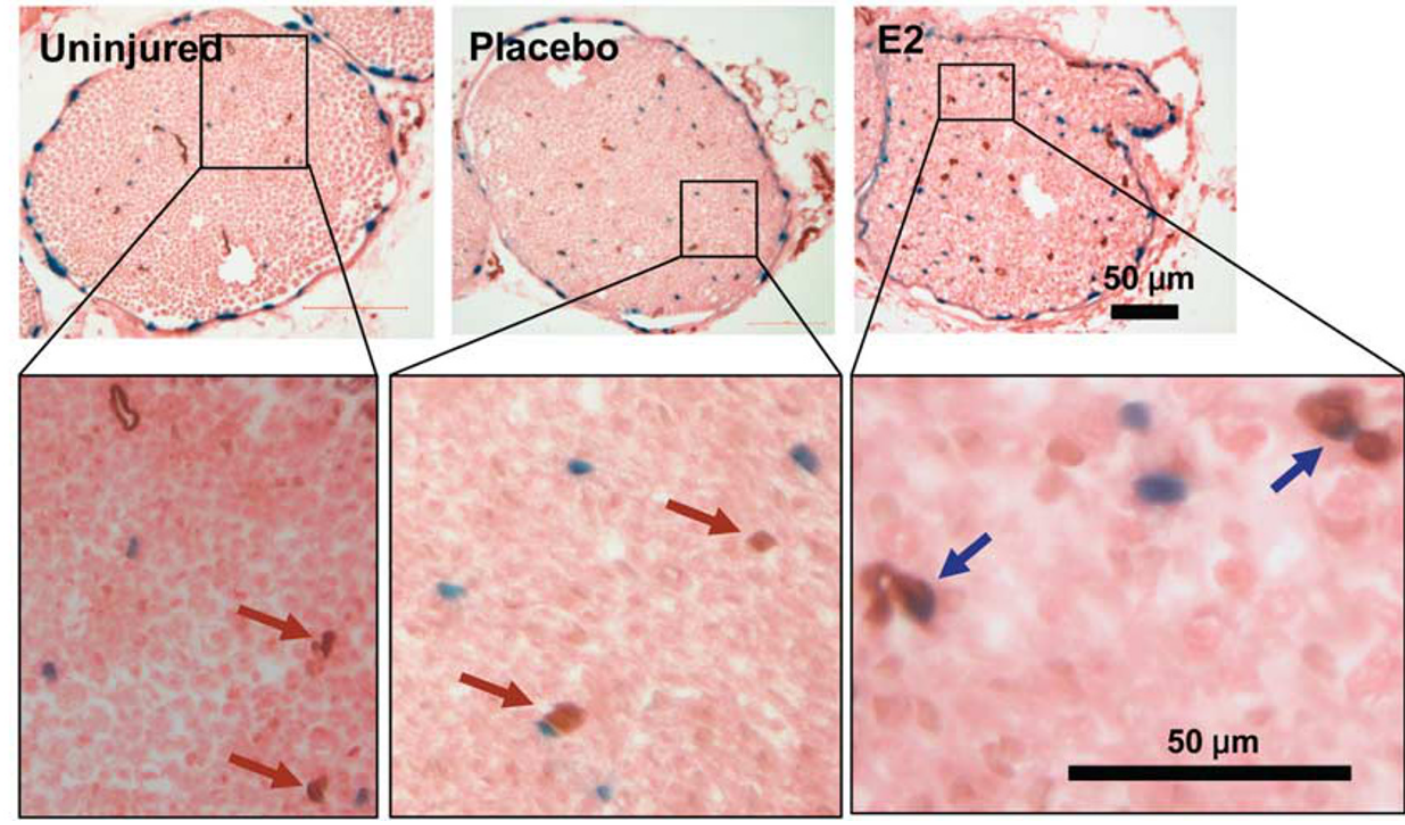


administered both E2 and cyclopamine was accompanied by a decline in Shh signaling.

\section{E2 Downregulates the Expression of HIP after Nerve- Crush Injury}

To determine whether Shh-pathway activation after nervecrush injury could be triggered by a decline in expression of the Shh-inhibitor HIP, and whether E2 enhances HIP downregulation, HIP mRNA expression was monitored in the injured and uninjured contralateral nerves of placebo-treated and E2-treated mice. In placebo-treated nerves, HIP expression declined during the first $12 \mathrm{~h}$ after injury and then increased through (at least) day 7 , when it exceeded non-injury levels by nearly 3 -fold. In E2-treated nerves, the decline was similar, but the increase was slowed: HIP levels on day 7 were similar to non-injury levels and approximately 63\% lower than the levels measured in injured, placebo-treated nerves (Figure 5a). These observations were corroborated by assessments performed in mice treated with both E2 and ICI: 3 days after injury, HIP expression in placebo-treated nerves and in nerves treated with both E2 and ICI was similar and significantly higher than HIP expression in nerves treated with E2 alone (Placebo $v s$ E2: $P<0.01$; E2 + ICI vs E2: $P<0.03$; Figure $5 \mathrm{~b}$ ).

To determine which cells in the nerve express HIP, sections of nerve tissue were fluorescently immunostained for HIP expression and for co-expression of HIP and CD31 or HIP and S100 (a Schwann-cell marker). HIP expression was observed in both endothelial cells and Schwann cells of uninjured nerves (Figure 5c), and the number of HIPexpressing cells was lower in E2-treated nerves than in placebo-treated nerves on day 3 after injury (Figure 5d); Ptch1 was also expressed by both cell types (Figure 5c). Collectively, these observations suggest that components of the Shh pathway are present in the endothelial cells and Schwann cells of uninjured nerves, but the activation of Shh signaling is inhibited by HIP.

\section{E2 does Not Alter the Expression of Shh-Pathway Components In Vitro, but Downregulates HIP Expression} To identify the direct effects of E2 on the expression of Shhpathway components, we determined whether E2 altered the mRNA expression of Shh, Ptch1, Gli1, or HIP in cultured HUVECs, fibroblasts (NIH 3T3), and Schwann cells (SW-10). E2 treatment did not significantly alter Shh (Figure 6a), Ptch1, or Gli1 (Supplementary Figure 2) expression in any of the cell lines; however, HIP expression in endothelial cells and
Schwann cells declined $(P<0.01$; Figure 6b). To determine whether the E2-induced decline in HIP expression could enhance Shh signaling in endothelial cells, HUVECs were treated with or without E2, and with or without Shh, and then Shh signaling was evaluated via the Gli-luciferase assay. Shh treatment alone did not increase luciferase activity, but E2 increased activity 2-fold, and co-treatment with E2 and Shh increased activity 3.6-fold (Figure 6c). Collectively, the results from our in-vivo and in-vitro expression assessments suggest that the beneficial effects of E2 after nerve-crush injury do not evolve from direct, E2-induced activation of Shh signaling; rather, E2 reduces HIP expression and, consequently, magnifies Shh signaling in response to injury.

\section{DISCUSSION}

The results presented here demonstrate that local E2 injection before nerve injury promotes functional recovery and vascular growth. MCV, the duration of exercise, and capillary density were higher in mice treated with local injections of E2 than in mice administered placebo treatment, and these benefits were not accompanied by significant increases in serum E2 levels, which could alleviate some of the concerns associated with hormone replacement therapy. The enhanced vascularity appeared to evolve through upregulated hedgehog signaling: the expression of Shh, the Shh receptor Ptch1, and the Shh transcriptional target Glil was higher in E2-treated mice than in placebo-treated mice, and the enhanced vascularity associated with E2 treatment was abolished by co-administration of cyclopamine, which blocks hedgehog signaling by interfering with smo. E2 also downregulated HIP expression on day 7 after injury and reduced HIP expression in cultured HUVECs and Schwann cells without altering Shh expression. Thus, E2 appears to enhance Shh signaling, at least in part, by downregulating HIP, particularly at later time points when Shh expression has returned to pre-injury levels.

Shh has an important role in nerve preservation during diabetic neuropathy, ${ }^{18,25}$ and Desert Hedgehog is expressed by Schwann cells and several other cell types in the peripheral nerves of adult mice. ${ }^{15}$ Thus, hedgehog signaling appears to function in adult neural cells, despite the apparent lack of Shh expression in uninjured peripheral nerves. Shh expression is induced immediately after sciatic nerve crush injury, as shown both here and in a previous report, ${ }^{17}$ which suggests that hedgehog signaling likely has an important role in the recovery of injured nerves. Nerve regeneration is crucially

Figure 4 E2-induced vessel growth in injured nerves requires Shh-pathway activation. (a-c) Ptch1-LacZ mice were injected with saline (placebo); with E2 (100 $\mu \mathrm{g}$ administered 1 week before sciatic nerve-crush injury); with cyclopamine (CYC) ( $50 \mathrm{mg} / \mathrm{kg}$ per day from 2 days before nerve-crush injury until the time of killing), which blocks hedgehog signaling by interfering with smo; or with both E2 and cyclopamine. (a) Functional vasculature was identified in sections of injured nerve tissue by injecting mice with fluorescein-conjugated BS-1 lectin 15 minutes before killing on day 28 after injury. (b) Capillary density was evaluated by staining cross sections of the vasa nervorum from mice killed on day 28 with the endothelial-cell marker CD31 and quantified. ${ }^{\star} P<0.01$ vs placebo, CYC, or E2 + CYC. (c) Ptch1 protein expression was evaluated in X-gal-stained whole-mount tissues from injured nerves, quantified, and presented as a percentage of the whole-nerve area. ${ }^{\star} P<0.05$ vs placebo, CYC, or E2 + CYC. (d) Gli1-LacZ mice were injected with saline (placebo), E2, cyclopamine, or both E2 and cyclopamine and sacrificed 28 days after sciatic nerve-crush injury; then, Gli1 protein expression was evaluated in X-gal-stained whole-mount tissues from the injured nerve, quantified, and presented as a percentage of the whole-nerve area. ${ }^{\star} P<0.05$ vs placebo, $C Y C$, or $E 2+C Y C$. 
dependent on angiogenesis, and Shh has been shown to induce angiogenesis after myocardial infarction in mice and swine, ${ }^{24}$ after ischemic hindlimb injury in mice, ${ }^{23}$ and in murine models of corneal angiogenesis ${ }^{26}$ and wound healing, ${ }^{27}$ perhaps by inducing expression of angiogenic factors such as VEGF, angiopoietin 1, and angiopoietin $2 .^{26}$

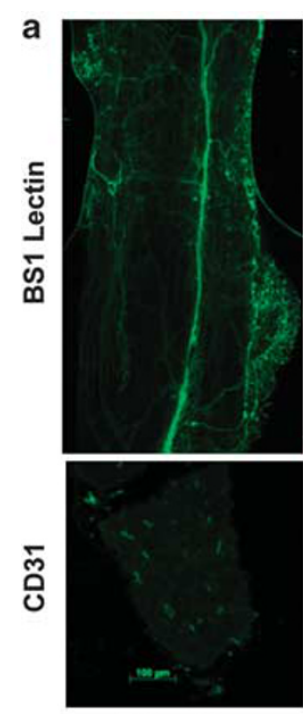

Placebo

C

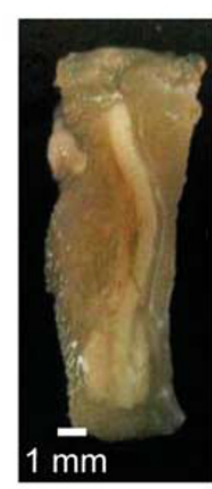

Placebo
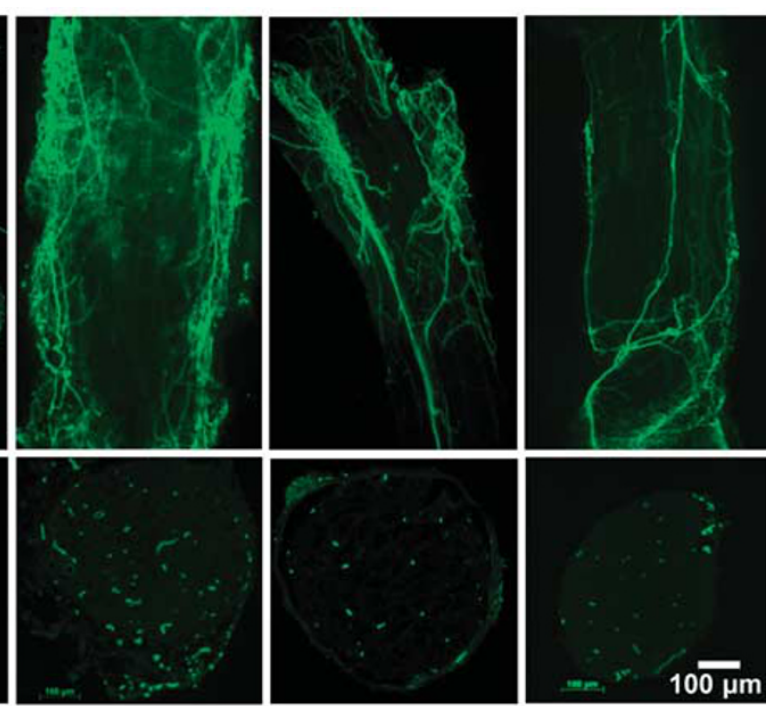

E2

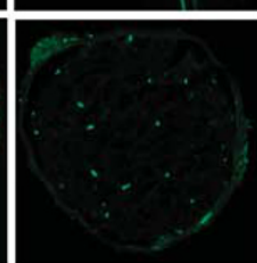

CYC

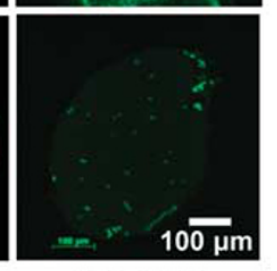

$\mathrm{E} 2+\mathrm{CYC}$

d

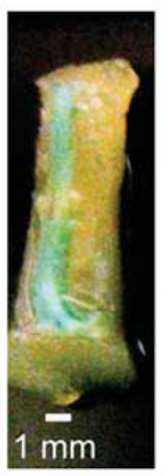

Placebo
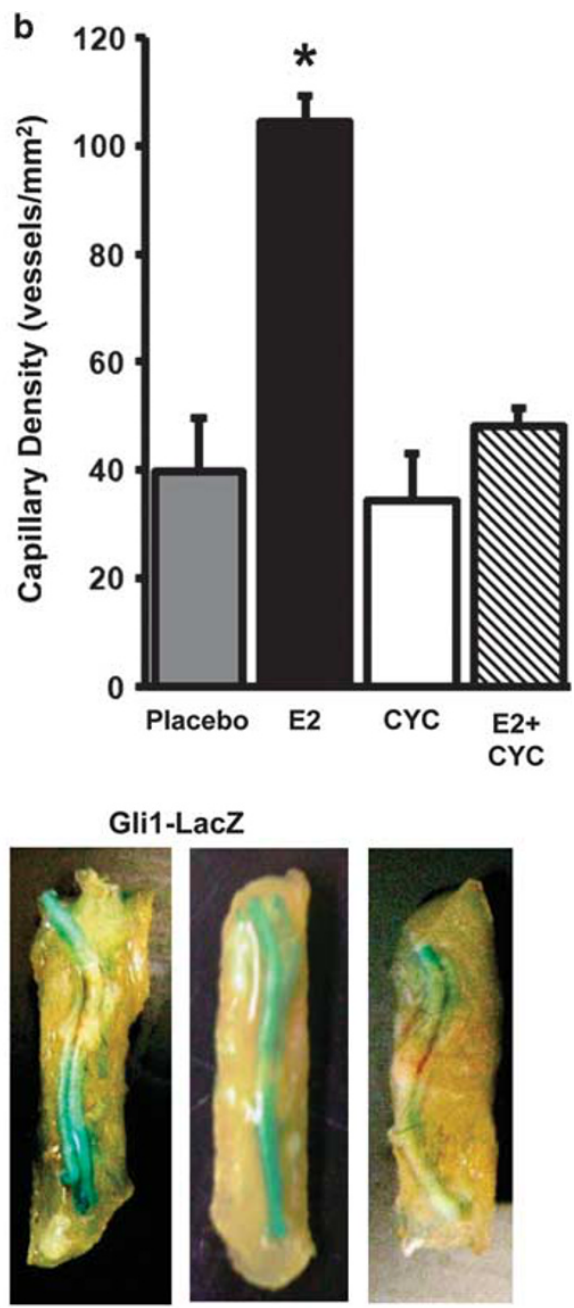

E2

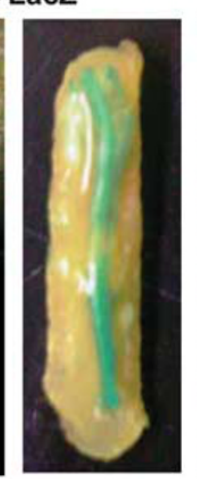

CYC

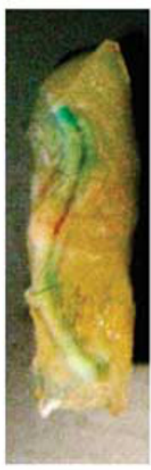

$E 2+C Y C$
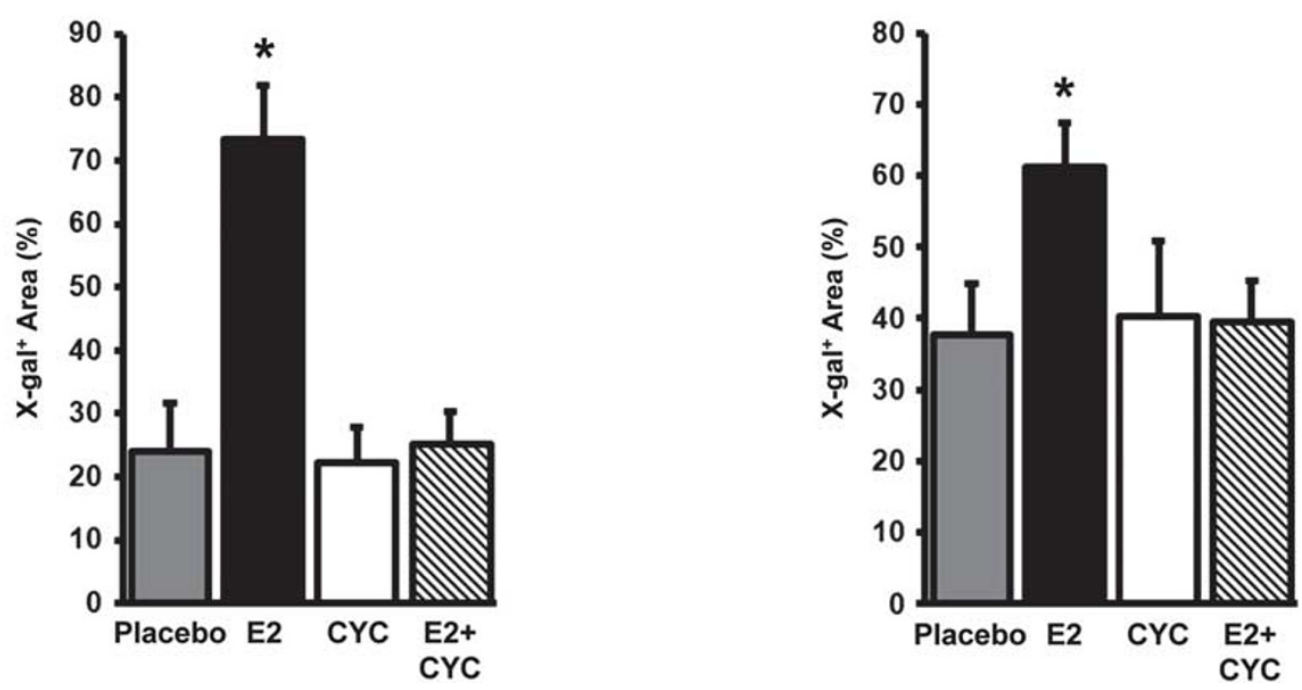
Shh is a secreted protein and can rapidly induce Gli1 or Ptch1 mRNA expression through an autocrine mechanism; however, Shh secretion may be more diffuse after injury, and at least some of the secreted protein likely enters the peripheral circulation. Both of these effects would reduce the local Shh concentration, which may explain why Gli1 and Ptch1 upregulation occurred considerably later (24-72 h after injury) than Shh upregulation (6h after injury) in our experiments. Fibroblasts respond strongly to Shh stimulation, ${ }^{27}$ but in endothelial cells the canonical Shh-induced activation of Gli1 does not occur, although endothelial cells express the Shh receptor Ptch1. Endothelial cells also express high levels of HIP, ${ }^{28}$ and the anti-angiogenic activity of HIP has been well documented by reports in the field of cancer a
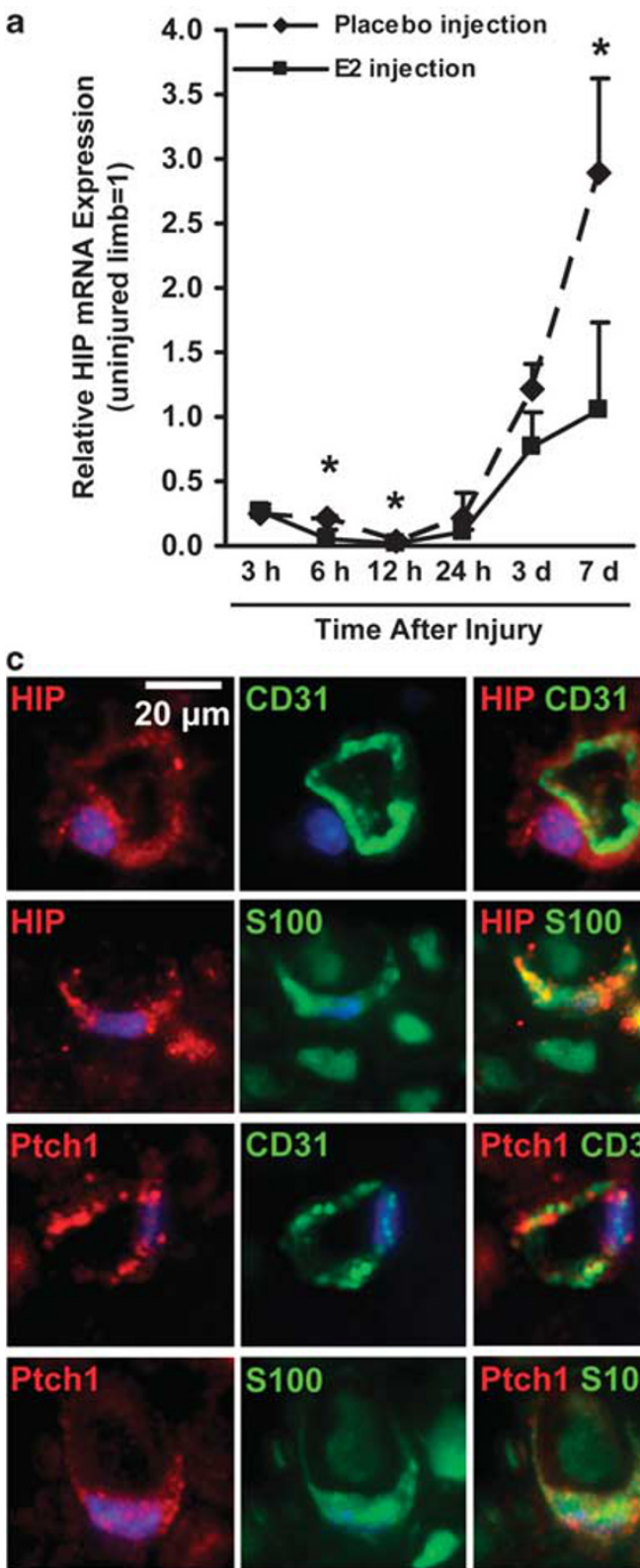
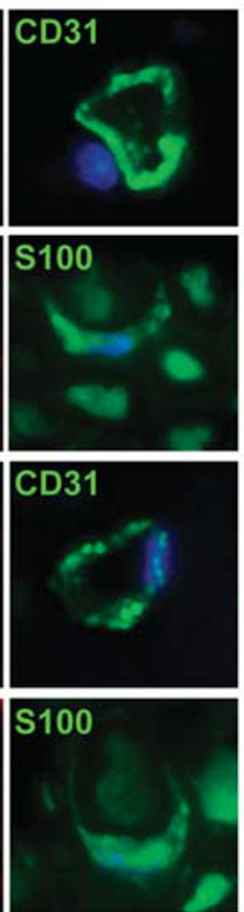

b

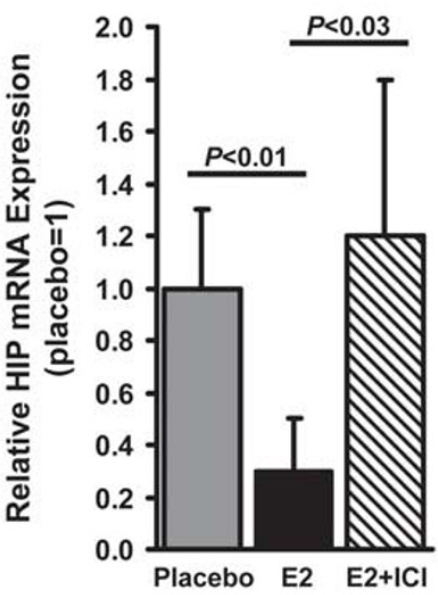

d
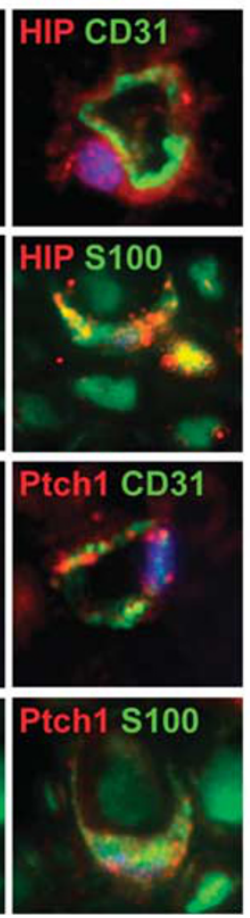
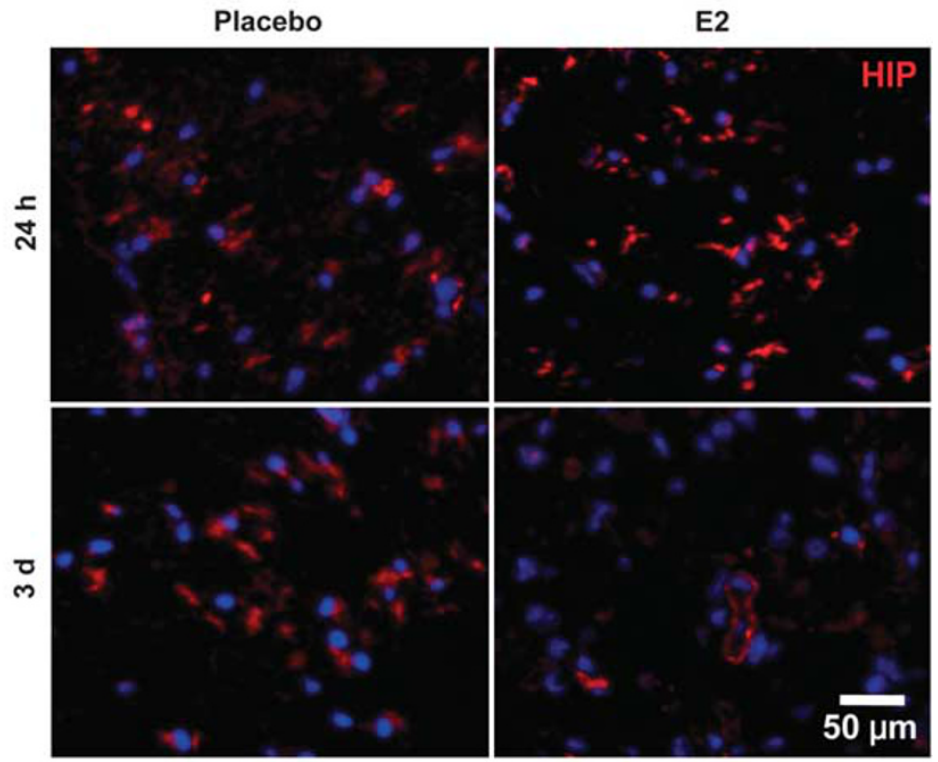

Figure 5 E2 downregulates HIP expression in injured nerves. (a) One week before surgical sciatic nerve-crush injury, E2 or saline (placebo) and PLGA was locally injected into the designated injury site, and the mRNA expression of HIP was evaluated from $3 \mathrm{~h}$ to 7 days after injury; measurements were performed via qRT-PCR, normalized to endogenous $18 \mathrm{~S}$ rRNA levels, and presented relative to the values obtained in the uninjured limb. (b) HIP mRNA expression was evaluated in mice treated with placebo, with E2 injections, or with injections of E2 and the E2-receptor blocker ICl; assessments were performed on day 3 after injury, when both Ptch1 and Gli1 mRNA expression were significantly upregulated (Figures 2c and d), via qRT-PCR, normalized to endogenous $18 \mathrm{~S}$ rRNA levels, and presented relative to the values obtained in placebo-treated mice. (c) Sections from uninjured nerves were stained for co-expression of (top row) HIP (red) and the endothelial-cell marker CD31 (green; second row) HIP (red) and the Schwann-cell marker S100 (green, third row) Ptch1 (red) and CD31 (green), or (bottom row) Ptch1 (red) and S100 (green); nuclei were counterstained with DAPI (blue). (d) Sections from placebo- and E2-treated mice killed $24 \mathrm{~h}$ or 3 days after injury were stained for HIP expression (red); nuclei were counterstained with DAPI (blue). ${ }^{\star P}<0.01$. 

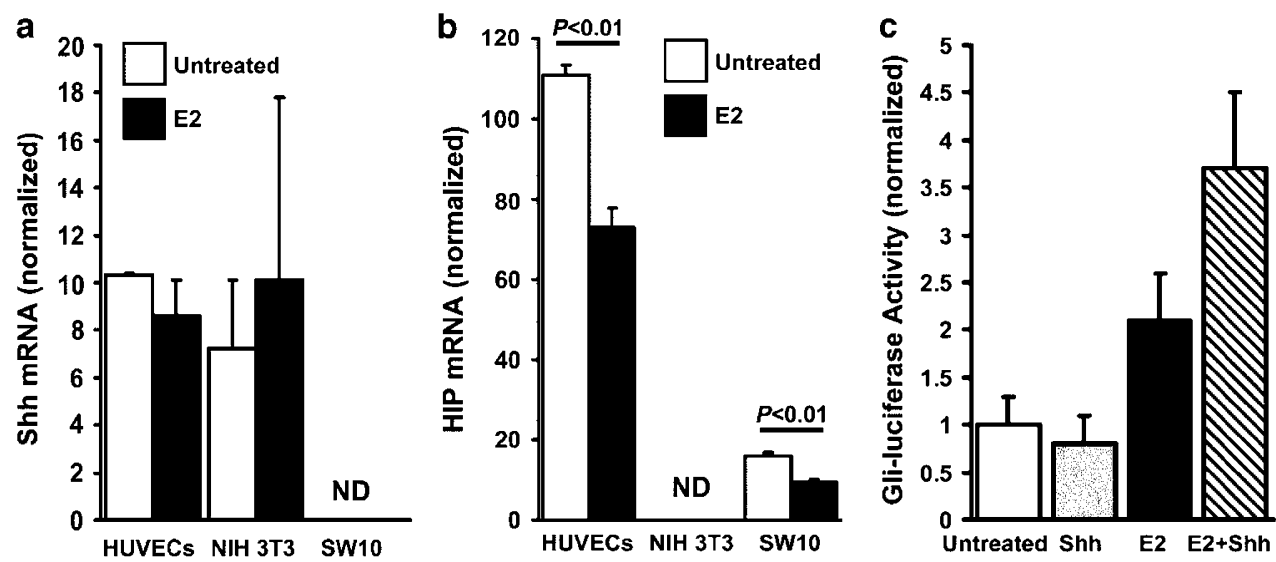

Figure 6 E2 enhances Shh signaling in endothelial cells by downregulating HIP expression. (a) Shh and (b) HIP mRNA expression were evaluated in HUVECs, fibroblasts (NIH 3T3), and Schwann cells (SW10) that had been treated with $1 \times 10^{-8} \mathrm{~mol} / \mathrm{I}$ E2 for $6 \mathrm{~h}$; measurements were performed via qRT-PCR and normalized to endogenous $18 \mathrm{~S}$ rRNA levels. (c) HUVECs were treated with or without E2 (6 h) and with or without $1 \mu \mathrm{g} / \mathrm{ml} \mathrm{Shh} \mathrm{(16h),} \mathrm{and} \mathrm{then} \mathrm{Shh} \mathrm{signaling}$ was evaluated via the Gli-luciferase assay. ND indicates not detectable.

research. ${ }^{28,29}$ HIP is a type 1 membrane-associated protein that impedes hedgehog signaling by binding and sequestering hedgehog proteins both at the cell surface and extracellularly; ${ }^{16,30-33}$ consequently, ectopic expression of HIP inhibits hedgehog signaling, whereas declines in HIP expression enhance hedgehog signaling. ${ }^{30}$ E2 treatment downregulated HIP expression in endothelial cells and Schwann cells, and Shh signaling in cultured endothelial cells was activated by treatment with E2 and (especially) E2 and Shh, but not by treatment with Shh alone. Thus, E2 likely increases Shh signaling in endothelial cells both intracellularly and in response to Shh produced by neighboring fibroblasts or Schwann cells, thereby increasing the contribution of endothelial cells to vascular growth.

VEGF levels after nerve injury were approximately 3-fold higher in E2-treated nerves than in placebo-treated nerves. Estrogen treatment also enhanced VEGF expression in a murine myocardial infarction model ${ }^{34}$ and increased the mobilization of bone-marrow-derived progenitor cells after myocardial infarction ${ }^{34}$ and arterial injury, ${ }^{35}$ perhaps by increasing expression of endothelial nitrous oxide synthase in the bone marrow. ${ }^{35}$ Collectively, these observations suggest that the pro-angiogenic properties of E2 could evolve, at least in part, through increases in VEGF expression and (possibly) progenitor-cell mobilization, and that E2-induced Shh signaling after nerve injury may be linked to signaling by VEGF and/or endothelial nitrous oxide synthase. Confirmation of these potential mechanisms requires additional investigation.

In conclusion, our findings demonstrate that local E2 injection before nerve injury promotes functional recovery and vascular growth afterward, and these benefits appear to evolve, at least in part, through E2-induced declines in HIP levels and a resultant upregulation of hedgehog signaling. Furthermore, local E2 injection did not significantly increase serum E2 levels, which could alleviate some of the concerns associated with hormone replacement therapy, such as elevated risk of breast cancer. Thus, local administration of an extended-release E2 preparation may be a viable strategy for enhancing angiogenesis in injured neuronal tissue.

Supplementary Information accompanies the paper on the Laboratory Investigation website (http://www.laboratoryinvestigation.org)

\section{ACKNOWLEDGEMENTS}

We thank W. Kevin Meisner, PhD, ELS, for editorial support and Kari Krueger for administrative assistance. This work was supported by the following Grants from the US National Institutes of Health: HL-53354, HL-57516, HL77428, HL-63414, HL-80137, HL95874, PO1HL-66957.

\section{DISCLOSURE/CONFLICT OF INTEREST}

The authors declare no conflict of interest.

1. Salpeter SR, Cheng J, Thabane L, et al. Bayesian meta-analysis of hormone therapy and mortality in younger postmenopausal women. Am J Med 2009;122:1016-1022 e1011.

2. Stampfer MJ, Colditz GA, Willett WC, et al. Postmenopausal estrogen therapy and cardiovascular disease. Ten-year follow-up from the nurses' health study. N Engl J Med 1991;325:756-762.

3. Rossouw JE, Anderson GL, Prentice RL, et al. Risks and benefits of estrogen plus progestin in healthy postmenopausal women: principal results From the Women's Health Initiative randomized controlled trial. JAMA 2002;288:321-333.

4. Salpeter SR, Walsh JM, Greyber E, et al. Brief report: Coronary heart disease events associated with hormone therapy in younger and older women. A meta-analysis. J Gen Intern Med 2006;21:363-366.

5. Hsia J, Langer RD, Manson JE, et al. Conjugated equine estrogens and coronary heart disease: the Women's Health Initiative. Arch Intern Med 2006;166:357-365.

6. Johns A, Freay $A D$, Fraser $W$, et al. Disruption of estrogen receptor gene prevents 17 beta estradiol-induced angiogenesis in transgenic mice. Endocrinology 1996;137:4511-4513.

7. Krasinski K, Spyridopoulos I, Asahara T, et al. Estradiol accelerates functional endothelial recovery after arterial injury. Circulation 1997;95:1768-1772.

8. Islamov RR, Hendricks WA, Katwa LC, et al. Effect of 17 beta-estradiol on gene expression in lumbar spinal cord following sciatic nerve crush injury in ovariectomized mice. Brain Res 2003;966:65-75.

9. Islamov RR, Hendricks WA, Jones RJ, et al. 17Beta-estradiol stimulates regeneration of sciatic nerve in female mice. Brain Res 2002; 943:283-286 
10. Wilson ME, Liu Y, Wise PM. Estradiol enhances Akt activation in cortical explant cultures following neuronal injury. Brain Res Mol Brain Res 2002;102:48-54.

11. Nusslein-Volhard C, Wieschaus E. Mutations affecting segment number and polarity in Drosophila. Nature 1980;287:795-801.

12. Roelink $\mathrm{H}$, Augsburger A, Heemskerk J, et al. Floor plate and motor neuron induction by vhh-1, a vertebrate homolog of hedgehog expressed by the notochord. Cell 1994;76:761-775.

13. Wang $M$, Jin P, Bumcrot DA, et al. Induction of the dopaminergic neuron phenotype in the midbrain by sonic hedgehog protein. Nature Med 1995;1:1184-1188.

14. Chiang C, Litingtung $Y$, Lee $E$, et al. Cyclopia and defective axial patterning in mice lacking Sonic hedgehog gene function. Nature 1996;383:407-413.

15. Parmantier E, Lynn B, Lawson D, et al. Schwann cell-derived Desert hedgehog controls the development of peripheral nerve sheaths. Neuron 1999;23:713-724.

16. Chuang PT, McMahon AP. Vertebrate Hedgehog signalling modulated by induction of a Hedgehog-binding protein. Nature 1999;397:617-621.

17. Hashimoto M, Ishii K, Nakamura Y, et al. Neuroprotective effect of sonic hedgehog up-regulated in Schwann cells following sciatic nerve injury. J Neurochem 2008;107:918-927.

18. Kusano KF, Allendoerfer KL, Munger W, et al. Sonic hedgehog induces arteriogenesis in diabetic vasa nervorum and restores function in diabetic neuropathy. Arterioscler Thromb Vasc Biol 2004;24:2102-2107.

19. Koga K, Nakamura $M$, Nakashima $H$, et al. Novel link between estrogen receptor alpha and hedgehog pathway in breast cancer. Anticancer Res 2008;28:731-740.

20. Institute of Laboratory Animal Resources Commission on Life Sciences National Research Council. Guide for the Care and Use of Laboratory Animals. National Academy Press: Washington, DC, 1996.

21. Andersson $\mathrm{N}$, Islander $\mathrm{U}$, Egecioglu $\mathrm{E}$, et al. Investigation of central vs peripheral effects of estradiol in ovariectomized mice. J Endocrinol 2005;187:303-309.

22. De Koning $\mathrm{P}$, Brakkee JH, Gispen WH. Methods for producing a reproducible crush in the sciatic and tibial nerve of the rat and rapid and precise testing of return of sensory function. Beneficial effects of melanocortins. J Neurol Sci 1986;74:237-246.

23. Pola R, Ling LE, Aprahamian TR, et al. Postnatal recapitulation of embryonic hedgehog pathway in response to skeletal muscle ischemia. Circulation 2003;108:479-485.
24. Kusano KF, Pola R, Murayama $\mathrm{T}$, et al. Sonic hedgehog myocardial gene therapy: tissue repair through transient reconstitution of embryonic signaling. Nat Med 2005;11:1197-1204.

25. Calcutt NA, Allendoerfer KL, Mizisin AP, et al. Therapeutic efficacy of sonic hedgehog protein in experimental diabetic neuropathy. J Clin Invest 2003;111:507-514.

26. Pola R, Ling LE, Silver $M$, et al. The morphogen Sonic hedgehog is an indirect angiogenic agent upregulating two families of angiogenic growth factors. Nat Med 2001;7:706-711.

27. Asai J, Takenaka $\mathrm{H}$, Kusano KF, et al. Topical sonic hedgehog gene therapy accelerates wound healing in diabetes by enhancing endothelial progenitor cell-mediated microvascular remodeling. Circulation 2006;113:2413-2424.

28. Olsen $\mathrm{CL}$, Hsu PP, Glienke J, et al. Hedgehog-interacting protein is highly expressed in endothelial cells but down-regulated during angiogenesis and in several human tumors. BMC Cancer 2004;4:43.

29. Kayed H, Kleeff J, Esposito I, et al. Localization of the human hedgehog-interacting protein (Hip) in the normal and diseased pancreas. Mol Carcinog 2005;42:183-192.

30. Chuang PT, Kawcak T, McMahon AP. Feedback control of mammalian Hedgehog signaling by the Hedgehog-binding protein, Hip1, modulates Fgf signaling during branching morphogenesis of the lung. Genes Dev 2003;17:342-347.

31. Kawahira H, Ma NH, Tzanakakis ES, et al. Combined activities of hedgehog signaling inhibitors regulate pancreas development Development 2003;130:4871-4879.

32. Treier M, O'Connell S, Gleiberman A, et al. Hedgehog signaling is required for pituitary gland development. Development 2001; 128:377-386.

33. Coulombe J, Traiffort E, Loulier $\mathrm{K}$, et al. Hedgehog interacting protein in the mature brain: membrane-associated and soluble forms. Mol Cell Neurosci 2004;25:323-333.

34. Hamada $\mathrm{H}$, Kim MK, Iwakura $\mathrm{A}$, et al. Estrogen receptors alpha and beta mediate contribution of bone marrow-derived endothelial progenitor cells to functional recovery after myocardial infarction. Circulation 2006;114:2261-2270.

35. Iwakura A, Luedemann C, Shastry S, et al. Estrogen-mediated endothelial nitric oxide synthase-dependent mobilization of bone marrow-derived endothelial progenitor cells contributes to reendothelialization after arterial injury. Circulation 2003;108: 3115-3121. 